\title{
Robotic Additive Manufacturing with Toy Blocks
}

\author{
Mikiya Kohama $^{a *}$, Chiharu Sugimoto $^{\mathrm{b}}$, Ojiro Nakano ${ }^{\mathrm{b}}$ and Yusuke Maeda ${ }^{\mathrm{c}}$ \\ ${ }^{a}$ Specialization in Mechanical Engineering, Dept. of Mechanical Engineering, \\ Materials Science, and Ocean Engineering, Graduate School of Engineering Science, \\ Yokohama National University, Kanagawa, Japan, kohama-mikiya-ry@ynu.jp \\ ${ }^{b}$ Specialization in Mechanical Engineering, Dept. of Systems Integration, Graduate \\ School of Engineering, Yokohama National University, Kanagawa, Japan \\ ${ }^{c}$ Div. of Systems Research, Faculty of Engineering, Yokohama National University, \\ Kanagawa, Japan
}

\section{Biography}

Mikiya Kohama received the B. E. degree from Yokohama National University in 2019.

Chiharu Sugimoto received the M. E. degree from Yokohama National University in 2019.

Ojiro Nakano received the M. E. degree from Yokohama National University in 2016.

Yusuke Maeda received the B.E., M.E. and D. Eng. degrees from The University of Tokyo in 1995, 1997 and 2003, respectively. From 1997 to 1999, he worked in Dai Nippon Printing Co., Ltd. From 1999 to 2004, he was a Research Associate at Department of Precision Engineering, The University of Tokyo. He joined Division of Systems Research, Faculty of Engineering, Yokohama National University as a Lecturer in 2004. From 2005, He is an Associate Professor of the division. His research interests include robotic manipulation, robot programming, and decentralized manufacturing systems. Dr. Maeda is a member of RSJ, JSME, JSPE, SICE and IEEE-RAS. 


\title{
Robotic Additive Manufacturing with Toy Blocks
}

\author{
Abstract \\ We study a block type 3D printer that performs assembly of toy block from 3D \\ CAD models. In this system, a 3D CAD model is automatically converted to a \\ block model consisting of primitive shapes of toy blocks. Then a feasible \\ assembly plan of the block model is also generated automatically. According to \\ the assembly plan, an industrial robot assembles a block sculpture layer by layer \\ from bottom to top. This approach has advantages in terms of reusability of \\ materials and easiness of combining multiple materials, which are difficult for \\ conventional 3D printers. We introduce a technique to determine the order of \\ block placement to assemble various patterns of block models reliably. The \\ technique includes converting unassemblable shapes in the model to assemblable \\ ones with support blocks and/or decomposition into subassemblies. In addition to \\ this, we implement a robot control system to automatically generate a desired \\ sculpture stably according to the determined placement order. Experiments of \\ assembling toy block models are demonstrated. The results show that our system \\ can assemble various block models.
}

Keywords: additive manufacturing, digital fabrication, digital material, automatic assembly, industrial robot, block printing.

\section{Introduction}

Nowadays 3D printers become popular. Additive manufacturing (AM), which is basis of 3D printers, is a process that generates a desired shape by adding materials incrementally. AM can generate a shape directly from a design. It enables us to generate a shape that cannot be realized by using conventional techniques such as removal machining. Nowadays the evolution of AM is phenomenal and it would result in a fast transition from traditional methods of manufacturing to AM in the near future (Ngo et al., 2018). A typical 3D printer, using FDM (fused deposition modelling) for example, laminates materials continuously. It can easily generate complex shapes and perform one-piece molding; however, it has difficulty in dealing with multiple materials simultaneously. In addition, it has a limited range of available materials (Langford et 
al., 2016). Moreover, we have to choose an appropriate printer for the material.

Since various shapes can be generated by assembling standardized digital materials, block type or voxel type 3D printers are studied (Hiller and Lipson, 2009A). We proposed a robotic block printing system that automatically assembles a block sculpture (Maeda et al., 2016; Sugimoto et al., 2017). First, the system converts a 3D CAD model into a block model. Secondly, a feasible order of block placement is decided. Finally, a robot assembles each block according to the determined order and the desired shape is "printed."

This approach naturally has a limitation in terms of resolution of assembled shapes due to the size of digital materials. However, our block printing system has some advantages over conventional 3D printers. Our approach makes it possible to construct objects composed of multiple materials easily. Color printing is also possible with colored blocks. In addition, since our approach does not use any adhesive during assembly, generated shapes can be disassembled into the smallest unit. As a matter of course, the disassembled components are reusable as materials for another shape.

In our previous studies, some preliminary results on the block printing system were reported (e.g., Maeda et al., 2016; Sugimoto et al., 2017). In this paper, we present our improved system that can assemble various block models more reliably and more rapidly with optimization of robot motion.

\section{Related Works}

Several groups have studied 3D printing with digital materials. Hiller and Lipson analyzed "voxel printing" in detail (Hiller and Lipson, 2009A). Additionally, they assembled voxel models by adhering voxels (Hiller and Lipson, 2009B). They showed that voxel printing has some advantages including perfect repeatability and multiple materials. Medellin et al. automatically assembled a voxel model by adhering voxels (Medellin et al., 2010). Their technique made it possible to assemble an unstable shape by adding support voxels around the unstable voxels. However, due to requirement of adhering for assembly, the techniques above lack reusability of sculptures. Sekijima et al. proposed a digital material named "Kelvin block," 
which connects each other by magnetic force (Sekijima et al., 2015). Due to its magnetic force and space-filling shape, Kelvin blocks can correct the positional errors during automatic assembling. However, since this technique requires magnetic blocks, there is limitation of diversity of blocks. Suzuki et al. presented an automatic assembly system using Dynablock, their original magnetic block (Suzuki et al., 2018). Since the system deals with dozens of blocks simultaneously, it can assemble desired block models quite rapidly. On the other hand, as the system is specific to Dynablock, the system cannot deal with multiple materials. Dynablock itself is complex an not easy to produce. Gilday et al. proposed a technique of automatic assembling, disassembling and reassembling LEGO block models (Gilday et al., 2018). Their approach can optimize a block operation order in terms of time, number of block manipulation and success probability; however, it does not consider mechanical stability of block sculptures during operations. Additionally, their technique substantially limits a range of printable sculpture because it carries a block by grasping its side surfaces; this grasping cannot place a block where its adjacent space is already occupied. In contrast to the previous studies, we present a 3D block printer that can assemble various shapes with simple mass-producible blocks without adhesion.

Some studies explored the possibility of additive manufacturing with digital materials. Mueller et al. proposed an assembly technique combining block printing and conventional 3D printing (Mueller et al., 2014). In the technique, a block model is assembled manually as close as possible to the desired model. Then the remaining regions are generated by conventional 3D printing. Printing time by this technique is much shorter than that by only a conventional 3D printer. It can also cope with the limitation of block printing in terms of shape resolution. MacCurdy et al. designed blocks for assembling electromechanical structures (MacCurdy et al., 2014). Each block has multiple connections and a simple electrical function such as resistance, battery and actuator. Various electromechanical devices can be realized by assembling these blocks. Langford et al. developed a system that assembles some kinds of electronic components such as capacitors and inductors (Langford et al., 2016). These components were made by 
combining conductor blocks and insulator blocks. The studies mentioned above show potential of block printing.

\section{Building Blocks}

We use "nanoblock" by Kawada as building blocks for additive manufacturing. The toy blocks have LEGO-like shapes with studs (figure 1(a)). The smallest block has size $4 \mathrm{~mm}(\mathrm{~W}) \times 4 \mathrm{~mm}$ (D) $\times 3 \mathrm{~mm}(\mathrm{H})$. In this paper, we denote the horizontal unit length $(4 \mathrm{~mm})$ by 1 unit. The block size is denoted by the number of rows $\times$ columns of its studs. We use $1 \times 1,1 \times 2,2 \times 1$ and $2 \times$ 2 nanoblock with the minimum height $(3 \mathrm{~mm}$ ) as primitive building blocks (figure $1(\mathrm{~b})) .1 \times 2$ blocks can be transposed to $2 \times 1$ by rotating them 90 degrees; however, $1 \times 2$ and $2 \times 1$ blocks are distinguished during an assembly planning. Our system uses seven colors for each type of the blocks for color printing. It also uses translucent blocks as support blocks (figure 1(c)) during automatic assembly in order to improve assemblability.

We assemble the building blocks layer by layer from bottom to top using an industrial robot. The layers are numbered from 1 (the bottom) to $n$ (the top).

\section{Conversion from 3D CAD Models to Block Models}

First, we use an algorithm to convert 3D CAD models to voxel models. A 3D CAD model in STL format is converted to a set of voxels. We currently operate the procedure using a web service for decomposition into cubic voxels (Arjan, 2019). We modify the height of a CAD model 4/3 times in advance because $1 \times 1$ nanoblock is not cubic (4(W):4(D):3(H)). After that, we determine the arrangement of blocks to generate a block model so that the block model has identical shape to the voxel model. The arrangement is executed in the following steps:

(1) Initialize a list of the available block sizes: $L=\{2 \times 2,2 \times 1,1 \times 2,1 \times 1\}$.

(2) Dequeuer the first element $s$ from $L$.

(3) Initialize a variable: $k=0$

(4) Fulfill the $k$-th layer of the voxel model by the blocks whose size is $s$. 
(5) While $k<n$, increment $k$ and repeat step 4 (proceed to the next layer).

(6) Repeat step 2 to step 5 unless $L$ is empty.

In step 4, the reference point for the arrangement is shifted one unit in both row and column directions layer by layer in order to increase the number of block-to-block connections (figure 2). In our current implementation, finally, we give the colors to each block manually. However, it is not difficult to assign the colors automatically if a colored 3D CAD model is given (e.g., Kozaki et al., 2016).

\section{Assembly Planning}

\subsection{Assembly Failures}

In order to illustrate the importance of assembly planning, we describe two patterns of assembly failures. The first one is very simple; it is impossible to assemble a floating block (figure 3(a)) because our system assembles block models from bottom to top. To describe the other pattern, let us consider placing blocks in the $k$-th layer. As shown in the left case of figure 3(b), when we try to place the blocks in the order of $\mathrm{A}$ and $\mathrm{B}$, the red block in the $(k-1)$-th layer would rotate in the process of placement. This leads to an assembly failure. Note that this rotation is unavoidable even under perfect position control of block A because the force required to insert block A into the studs is larger than the maximum force that the red block can resist. On the other hand, as shown in the right case of figure 3(b), when we try to place the blocks in the order of B and A, the placement of block B reinforces the scaffold including the red block. Therefore, the insertion of block A afterward does not cause the rotation of the red block and assembly succeeds.

These examples demonstrate that the order of block placement must be taken into consideration in assembly planning. We describe the details of assembly planning below.

\subsection{Assemblability of Blocks}

Let us describe how to check the assemblability of a block in the $k$-th layer. The block must 
have one or more connections to studs of blocks in the $(k-1)$-th layer. We focus on the placement of a block in the $k$-th layer on a blocks in the $(k-1)$-th layer and introduce block assemblability as described below.

\subsubsection{Stable scaffold}

Here we focus on the space occupation by the block model. When we place the block $B$ in the $k$ th layer, we define $R_{k}$ as the region under $B$. If any of the following conditions is fulfilled, we label such placement "Case stable," the most stable situation: (i) $k=1$; (ii) $k=2$ and any space under $B$ is occupied by other blocks; (iii) $k>2$ and all spaces under $B$ are occupied by other blocks. Condition (i) indicates that $B$ is on the ground. Condition (ii) indicates that $B$ has one or more connections with on-ground blocks as shown in figure 4(a). (iii) means that there is no vacant space under $B$ as shown in figure 4(b).

\subsubsection{Contact Region Inclusion}

If the placement of block $B$ is not labelled as "Case stable," we proceed to the next check. We consider a connection between $B$ and one of the lower blocks, $L_{i}$ that connect with $B$. Suffix $i$, an index of connected lower blocks, is required because $B$ can have multiple connection to its lower layer. Here we focus on studs in the connection. Let the $1 \times 1$-size horizontal region corresponding to stud be denoted by $s$. We define $C_{k}$, the convex hull formed by $s$ of all the studs that connect $B$ and $L_{i}$. When $B$ has no connection with the lower layer, $C_{k}$ is an empty set. Additionally, we consider each connection between $L_{i}$ and its lower blocks. We define $\hat{C}_{k}^{i}$, the convex hull formed by $s$ of all the studs that connect $L_{i}$ and its lower blocks. We also define a projection operator $P$ that maps a region to the $\mathrm{X}-\mathrm{Y}$ plane. If the placement satisfies the following condition as shown in figure 5, we label it "Case inclusion," which is the secondly stable case:

$$
k>2 \bigwedge C_{k} \neq \varnothing \bigwedge P\left(C_{k}\right) \subseteq \bigcup_{i} P\left(\hat{C}_{k}^{i}\right)
$$


The left part of condition (1) indicates that $B$ connects with its lower layer, and the right part means that $B$ is supported by the $(k-1)$-th and $(k-2)$-th layer.

\subsubsection{Partial Contact Region Inclusion}

We define a map $A$ that returns the area of the specified region. If a placement is neither labelled "Case stable" nor "Case inclusion" but the following condition is fulfilled as shown in figure 6, we call the placement "Case partial inclusion," the thirdly stable case:

$$
k>2 \bigwedge \forall i A\left(P\left(C_{k}\right) \cap P\left(\hat{C}_{k}^{i}\right)\right)>0 .
$$

Condition (2) indicates that $B$ is partially supported by the $(k-1)$-th and $(k-2)$-th layer.

\subsubsection{Adjacent Support}

Let us consider the case as shown in figure 7(a). In this case, none of the conditions described above holds, and the green block in the $k$-th layer is not assemblable. However, after we place another adjacent block in the $k$-th layer as shown in figure $7(\mathrm{~b})$, it becomes possible to place the block that was not assemblable.

The change of the block assemblability is partially due to the stronger scaffold structure made by new connections between the blocks in the $(k-1)$-th layer through another block placement in the $k$-th layer. Moreover, the side contact between the blocks in the $k$-th layer also helps to avoid collapse of the structure. The rotation of the blocks in the $(k-1)$-th layer is accompanied by the rotation of the other block in the $k$-th layer, which is prevented by the side contact.

Thus we judge the situations like the case figure 7(b) as assemblable, where there is an adjacent block in the $k$-th layer that makes connection between the multiple blocks in the $(k-1)$-th layer under the placed block. We call such a case "Case adjacent support," which is the fourthly stable case. This case appears when the block $B$ has an adjacent block which connects with multiple lower blocks and one of them connects with $B$. 


\subsubsection{Unassemblable Cases}

If a connection does not fulfill any of the conditions described above, we consider it “Case unassemblable." An example of unassemblable cases is shown in figure 8(a). In this situation, the area of the intersection $P\left(C_{k}\right) \cap P\left(\hat{C}_{k}^{i}\right)$ is empty and there is no adjacent block that can support the green block. Another example is shown in figure 8(b). In this situation, the area of the intersection $P\left(C_{k}\right) \cap P\left(\hat{C}_{k}^{2}\right)$ is larger than 0 , but that of $P\left(C_{k}\right) \cap P\left(\hat{C}_{k}^{1}\right)$ is not. Thus, condition (2) does not hold and this situation is labelled "Case unassemblable." Note that a floating block also falls under this case. Such unassemblable cases must be overcome for 3D printing.

\subsection{Dealing with Unassemblable Shapes}

Since a robot assembles blocks from bottom to top layer by layer, blocks that have no stable scaffolds ("case unassemblable") cannot be assembled. For example, the floating red blocks in figure 9 cannot be assembled because they have no connections with the lower layer. We introduce two methods to deal with such unassemblable shapes: support blocks and subassemblies.

\subsubsection{Support Blocks}

One of the techniques to deal with unassemblable shapes is using support blocks. We can make unassemblable blocks assemblable by placing support blocks vertically under them like pillars. However, in our system, the support blocks must be removed manually after assembling. Thus, it is ideal that all support block pillars root to the ground. In some cases, however, support blocks are sandwiched between original component blocks as shown in the bottom of figure 9 . It takes time and effort to remove such interposed support blocks.

\subsubsection{Subassembly}

The other technique to deal with unassemblable shapes is dividing the original block model into subassemblies. Figure 10 is an example of division to subassemblies. The original block model 
is divided into upper and lower subassemblies. In contrast to adding support blocks, this approach requires no additional blocks to be removed. Therefore, shorter assembly time can be achieved. However, final joining of the subassemblies needs to be done manually. If one-layer subassemblies are generated, it is very troublesome to join them because their blocks have no mutual connections. Accordingly, we enforce the assembly planning algorithm not to generate one-layer subassemblies.

\subsection{Block Placement ordering}

In the previous subsections, we described assemblabilities of blocks and techniques to generate assemblable models. Those are used to determine the priority-based order of block placement. Here we define the following indices for each block in the $k$-th layer ("Block $B$ ") for block prioritization:

- $\quad n_{\text {block }}$ : the number of the blocks in the $(k-1)$-th layer with which $B$ connects.

- $n_{\text {stud }}$ : the number of the studs of the blocks in the $(k-1)$-th layer with which $B$ connects.

Figure 11 illustrates two examples of the indices. For the green block of figure 11(a), it is placed on two $2 \times 2$ blocks with one-stud connection for each. Thus $n_{\text {block }}=2$ and $n_{\text {stud }}=$ 2. In figure 11(b), the green block is placed on two $2 \times 2$ blocks with two-stud connection. Thus, $n_{\text {block }}=2$ and $n_{\text {stud }}=4$. Larger $n_{\text {block }}$ is preferable because the block placement on many blocks strengthens the scaffold structure, which may lead to changes of block assemblability (from "Case unassemblable" to "Case adjacent support"). Larger $n_{s t u d}$ is also preferable because a block placement on many studs leads to strong connection.

Let us describe the detail of the block placement ordering algorithm. The order is determined by the following steps:

(1) Initialize a placement-order list $L=\emptyset$ and a variable $k=1$.

(2) Initialize a temporary list $L_{k}=\varnothing$ 
(3) Determine the assemblability of each block in the $k$-th layer.

(4) Extract assemblable blocks that is not included in $L_{k}$, rearrange them in the order of assemblability, and then enqueue them to $L_{k}$. The ordering is executed by the following criteria (in order of descending):

○ Assemblability case is better ("stable"> "inclusion" > "partial inclusion"> "adjacent support").

○ $n_{\text {block }}$ is larger.

$\circ n_{\text {stud }}$ is larger.

- Position $x$ is smaller (to uniquely determine the assembly order).

- Position $y$ is smaller (to uniquely determine the assembly order).

(5) Repeat step (3) and (4) while we can add blocks to $L_{k}$. Note that block assemblabilities may change from "Case unassemblable" to "Case adjacent support" during these steps.

(6) If all blocks in the $k$-th layer are in $L_{k}$, add $L_{k}$ to $L$, and then go back to step 2 with $k \leftarrow$ $k+1$.

(7) Otherwise, if there are unassemblable blocks, fork to the following two plans.

(a) add support blocks in the $(k-1)$-th layer under all unassemblable blocks in the $k$-th layer, remove $L_{k-1}$ from $L$ and go back to step 2 with $k \leftarrow k-1$.

(b) divide the current model into two subassemblies so that the lower consists of 1 , $2, \ldots,(k-1)$-th layers and the upper consists of the other layers, add $L_{k}$ to $L$ and go back to step 2 with $k \leftarrow k+1$.

After these steps, a placement-order $L$, in other words, an assembly plan is given. However, due to the fork in step 7, multilple plans can be generated. It is inconvenience to wait for finishing planning and to select the best plan manually. Thus, our system reduces the number of plans by the following rules in step 7 of the planning algorithm: 
- All subassemblies in a plan must have at least $t_{\text {layer }}$ layers. Otherwise, the plan is immediately discarded.

- A plan can consist of $t_{\text {sub }}$ subassemblies at most. Otherwise, it is immediately discarded.

- A plan is discarded if we can find another plan that has fewer support blocks and consists of equal or fewer subassemblies.

$t_{\text {layer }}$ and $t_{\text {sub }}$ in the above rules are thresholds determined heuristically. These rules significantly reduce the number of assembly plans and planning time. We set $t_{\text {layer }}=2$ and $t_{\text {sub }}=3$. After assembly planning completes, the plans are divided into groups by their numbers of subassemblies. Then, for each group, a plan that contains the minimum support blocks is selected as its representative. Finally, we only need to choose the best plan among up to $t_{\text {sub }}$ representatives considering the balance between the number of support blocks and that of subassemblies. It is not difficult to automate assembly plan generation if we introduce a performance index to evaluate the balance.

\section{Robot Control System}

\subsection{System Overview}

To conduct assembly according to the assembly plan generated in section 5, a robot control system is required. We develop it by using ROS (Robot Operating System) (Quigley et al., 2009; Open Robotics, 2019) as a platform and MoveIt! (Willow Grage, 2019) as a motion planning software running on ROS. ROS aims to standardize functions of robotics. Nowadays many robots have interfaces between ROS and themselves. By using MoveIt!, we can generate trajectories that avoid collisions with objects around robots. Using such a standardized platform with collision-avoidance function, our system has compatibility with various robots including Cartesian, SCARA and six-axis robots and their environment. Moreover, MoveIt! can optimize trajectories of robots, which indicates that we can generate a desired block sculpture rapidly. 


\subsection{Force Control}

In the process of block assembly, each block is inserted to the studs of the scaffold blocks. If the scaffold structure is strong enough, the block insertion force should be large to make the inter-block connection tight. On the other hand, in some cases, large insertion forces may cause collapse of the scaffold. Thus the applicability of such large forces must be judged and insertion force must be controlled appropriately for each block.

In this study, the applicability of large insertion forces is checked based on block assemblability. If a block placement is labelled as "case stable," we judge that large insertion force is applicable. Otherwise, insertion force should be limited.

\subsection{Safer Block Path}

During block assembly, as shown in figure 12, sometimes we place a block where adjacent blocks (as the orange block in figure 12) already exist. If we place the block vertically in such a case, unexpected collision between the edge of the target block and that of its adjacent one may occur due to a warp of the sculpture or uncertainty of the posture of the carried block. To avoid this collision, firstly, we make an offset between the blocks as the left of figure 13 if the opposite space is vacant. Then the target block is moved obliquely to the position in the center of figure 13, where the surfaces of the blocks touch. Finally, a robot moves the block down vertically as the right of figure 13 . We can avoid unexpected collision between their edges by using this technique. Note that if both sides are occupied by other blocks, this technique cannot be applied. However, such a situation rarely appears due to our assembly order determination described in section 5.4. Assemblability cases of inner blocks tend to be "stable" and $n_{\text {block }}$ and $n_{\text {stud }}$ tend to be larger than those of outer blocks. It makes inner blocks be assembled prior to outer blocks. Thus there are few occasions to place a block whose both sides are occupied. 


\section{Experiments}

\subsection{Experimental Setup}

We prepared an experimental setup as shown in figure 14. A 6-axis industrial robot VP-6242G (Denso Wave) equipped with an electric gripper ESG-FS-2840 (Taiyo) was used. We designed fingers with grooves to grasp studs of a nanoblock (figure 15). We also designed a block supplier as shown in the right bottom of figure 14. In this supplier, when a robot picks a bottom block, the blocks stacked above drop and the next block is automatically loaded. We can refill blocks even during assembly. A plastic block plate with $20 \times 20$ studs was used as a ground of assembly.

A Linux PC with Core i7 3930K (3.20 [GHz]) was used to execute our block printing system. The system ran on Ubuntu 16.04LTS and the version of ROS was Kinetic. BiTRRT (Bi-directional Transition based Rapidly growing Random Tree) (Ahmed et al., 2017) was used as a motion planning algorithm in MoveIt!. Meijer et al. stated that this algorithm can optimize trajectories relatively fast (Meijer et al., 2017).

\subsection{Experimental Results}

We tested our block printing system with the models shown in figure 16-19. We used the CAD models that are available online for the experiments. The leftmost CAD models were converted to the block models. Then, their assembly plans were generated. Table 1 shows the conversion result of each model. The assembly plans were executed by the robot and the subassemblies containing support blocks were assembled. Finally, we removed the support blocks in the assembled model and joined the subassemblies manually in order to obtain the final assemblies.

Figure 20 shows a scene in assembly execution. All the models were successfully assembled. These results show that various models can be assembled even if they have fragile shapes such as overhang (the ears of the bunny) and a slender part (the tower) by using our approaches. Table 1 also shows the assembly time and speed and table 2 shows the assembly results of our previous study (Sugimoto et al., 2017). These results show that our new system is 
much faster than the previous system in assembly speed, but is slower in planning speed. The former is due to trajectory optimization by MoveIt!, and the latter is due to algorithm fork in step 7 of the assembly planning. Comparing the overall time (planning and assembly time), our new system generated the desired shapes much faster than the previous.

\section{Conclusion}

In this paper, we studied a block type 3D printer called "Block Printing System." We introduced the techniques of assembly planning and robot control for reliable assembly. The experimental results show that various block sculptures can be generated by using our system from their 3D CAD models.

In future work we can take several prospects into consideration. In terms of assembly planning, we should introduce mechanical analysis. Since our current assembly planning depends on empirical evaluation of assemblability, it is difficult to use building blocks of various sizes. Sugimoto and Maeda developed a technique to evaluate an assemblability of block mechanically (Sugimoto and Maeda, 2018), which would lead to reliable assembly planning with various blocks. We should also deal with larger blocks such as $4 \times 2$ or $8 \times 2$ in order to assemble larger scale models rapidly. Our techniques can apply to more practical use such as ceramic artificial bones (Oba et al., 2015). It can be also considered to fuse our system and a postprocessing method (e.g., additive or removal machining) to increase resolution of assembled block models.

\section{Acknowledgements}

This work was supported by the JSPS KAKENHI under Grant JP15H03944. 


\section{References}

Ngo, T., Kashani, A., Imbalzano, G., Nguyen, K. and Hui, D., (2018), “Additive manufacturing (3D printing): A review of materials, methods, applications and challenges," Composites Part B: Engineering, 143, 172-196.

Langford, W., Chassaei, A. and Gershenfeld, N., (2016), "Automated assembly of electronic digital materials," Proceedings of Manufacturing Science and Engineering Conf. (MSEC), 2, T01A013.

Hiller, J. and Lipson, H. (2009A), "Design and analysis of digital materials for physical 3D voxel printing," Rapid Prototyping Journal, 15(2), 137-149.

Maeda, Y., Nakano, O., Maekawa, T. and Maruo, S. (2016), "From CAD models to toy block sculptures: A 3D Block Printer," Proceedings of 2016 IEEE/RSJ Int. Conf. on Intelligent Robots and Systems (IROS), 2167-2172.

Sugimoto, C., Maeda, Y., Maekawa, T. and Maruo, S. (2017), “A 3D block printer using toy blocks for various models," Proceedings of 2017 13th IEEE Conference on Automation Science and Engineering (CASE), 958-963.

Hiller, J. and Lipson, H. (2009B), "Fully Recyclable multi-material printing," Proceedings of Solid Freeform Fabrication, 98-106.

Medellin, H., Corney, J., Ritchie, M. and Lim, T. (2010), "Fully recyclable multi-material printing," Solid Freedom and Manual Assembly Plans Using Octrees, 30(2), 23-26.

Sekijima, K., Masuda, T. and Tanaka, H., (2015), "Reconfigurable three-dimensional prototype system using digital materials," in Tran. of Virtual Reality Society of Japan, 20(2), 97105 (in Japanese).

Suzuki, R., Yamaoka, J., Leithinger, D. and Kakehi, Y., (2018), "Dynablock: dynamic 3D printing for instant and reconstructable shape formation," ACM User Interface Software and Technology (UIST), 99-111.

Gilday, K., Hughes, F. and Iida, F., (2018), “Achieving flexible assembly using autonomous robots and systems," Proceedings of IEEE/RSJ Int. Conf. on Intelligent Robots and Systems (IROS), 6105-6110.

Mueller, S., Mohr, T., Guenther, K., Frohnhofen, J. and Baudisch, P. (2014), “faBrickation: fast 3D printing of functional objects by integrating construction kit building blocks," Proceedings of 32nd annual ACM CHI Conf. on Human Factors in Computing System, $3827-3834$.

MacCurdy, R., McNicoll, A. and Lipson, H., (2014), "Bitblox: printable digital materials for electromechanical machines," The Int. Journal of Robotics Research, 33(10), 13421360. 
Arjan, W., "Voxelizer, convert your 3D model to voxels online." Accessed at drububu.com/miscellaneous/voxelizer/?out=jso, 2019.

Kozaki, T., Tedenuma, H. and Maekawa, T., (2016), "Automatic generation of LEGO building instructions from multiple photographic images of real objects," Computer-Aided Design, 70, 13 - 22.

Quigley, M., Gerkey, B., Conley, K., Faust, J., Foote, T., Leibs, E. and Berger, E. (2009), "ROS: an open source robot operating system," Proceedings of 2009 IEEE Int. Conf. on Robotics and Automation (ICRA). Accessed at https://www.willowgarage.com/sites/default/files/icraoss09-ROS.pdf, 2019.

Open Robotics, "ROS.org | Powering the world's robots." Accessed www.ros.org, 2019.

Willow Garage, "MoveIt Motion Planning Framework.” Accessed at https://moveit.ros.org, 2019.

Ahmed, S., Tan, Y., Lee, G., Chew, C. and Pang, C., (2016), “Object detection and motion planning for automated welding of tubular joints," Proceedings of IEEE/RSJ Int. Conf. on Intelligent Robots and Systems (IROS), 2610-2615.

Meijer, J., Lei, Q. and Wisse, M., (2017), "Performance study of single-query motion planning for grasp execution using various manipulators," Proceedings of Int. Conf. on Advanced Robotics (ICAR), 450-457.

Thingiverse (2019A), "Stanford Bunny by phooky.” Accessed at https://www.thingiverse.com/thing:3731, 2019.

Thingiverse (2019B), "Pixel tree topper star by knape.” Accessed at https://www.thingiverse.com/thing:194864, 2019.

Thingiverse (2019C), "Eiffel tower by Newcandle." Accessed at https://www.thingiverse.com/thing:912478, 2019.

Thingiverse (2019D), "Dog by YahooJAPAN.” Accessed at https://www.thingiverse.com/thing:182130, 2019.

Sugimoto, C., Maeda, Y., (2018), "Evaluation of assemblability by mechanical analysis in 3D block printer," 2018 JSME Conf. on Robotics and Mechatronics (ROBOMECH), 2A1H18 (in Japanese).

Oba, T., Maekawa, T., Maeda, Y. and Maruo, S., (2015), "Study on 3D block-printing: first report: fabrication of bioceramic blocks," Proceedings of the Symposium on MicroNano Science and Technology, 2015(7), 28pm3-B-7 (in Japanese). 
Table 1. Assembly results.

\begin{tabular}{c|c|c|c|c}
\hline & Bunny & Star & Tower & Dog \\
\hline $\begin{array}{c}\text { Number of blocks } \\
\text { (with support blocks) }\end{array}$ & 272 & 258 & 304 & 507 \\
Volume [unit] & 704 & 758 & 728 & 1266 \\
Number of support blocks & 25 & 14 & 46 & 24 \\
Number of subassemblies & 2 & 2 & 1 & 3 \\
Assembly planning time [s] & 13.42 & 11.29 & 6.16 & 56.05 \\
Assembling time [min] & 24.10 & 22.25 & 28.14 & 43.40 \\
Assembly speed [blocks/min] & 11.29 & 11.59 & 10.80 & 11.68 \\
\hline *1 unit =1 $\times 1$ block volume $=48 \mathrm{~mm}^{3}$ & & & \\
\hline
\end{tabular}

${ }^{*} 1$ unit $=1 \times 1$ block volume $=48 \mathrm{~mm}^{3}$.

Table 2. Assembly results of previous study (Sugimoto et al., 2017).

\begin{tabular}{c|c|c|c}
\hline & Bunny 1 & Bunny 2 & Duck \\
\hline Number of blocks & 281 & 262 & 565 \\
(with support blocks) & 31 & 12 & 44 \\
Number of support blocks & 2 & 5 & 2 \\
Number of subassemblies & 1.11 & 1.11 & 4.33 \\
Assembly planning time [s] & 38 & 34 & 134 \\
Assembling time [min] & 7.4 & 7.7 & 4.2 \\
Assembly speed [blocks/min] & & & \\
\hline
\end{tabular}




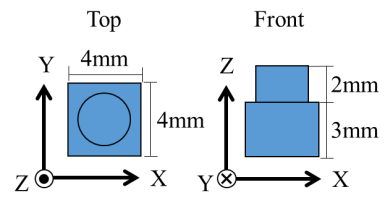

(a) Unit size

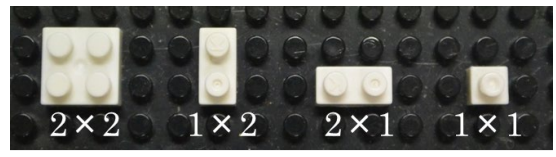

(b) Each size of nanoblock

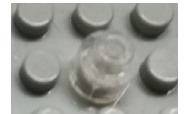

(c) Support

Figure 1. Nanoblocks.

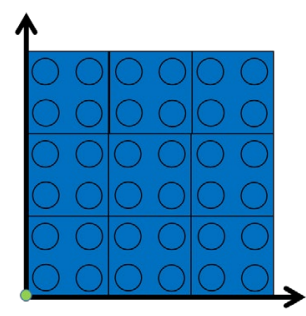

(a) Even layer

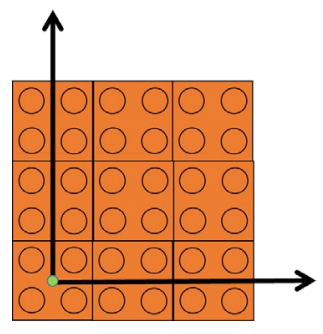

(b) Odd layer

Figure 2. Arrangement of blocks (Maeda et al., 2016).

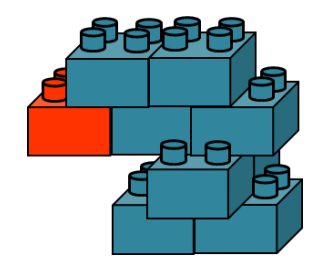

(a) The floating red block cannot be assembled.

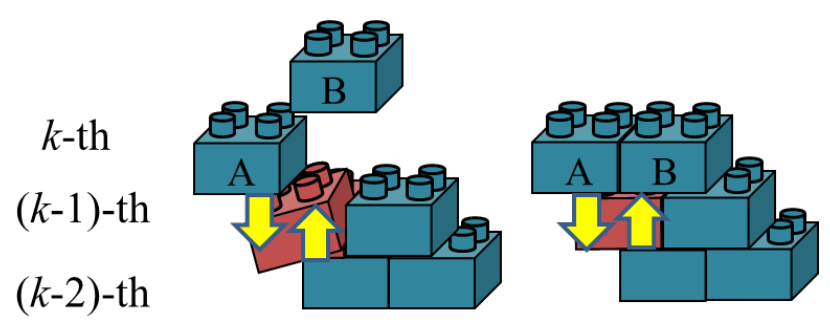

(b) Assembly order may lead an assembly failure. We must place block B before block A.

Figure 3. Assembly failure patterns. 


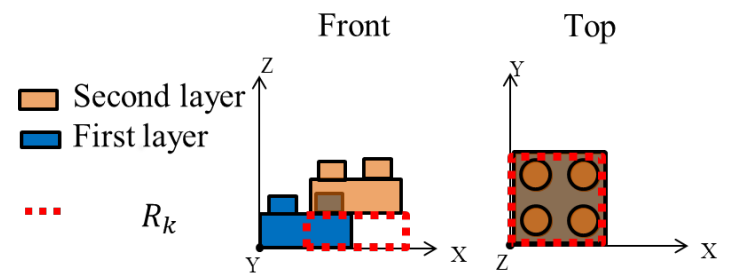

(a) The top block fulfills condition (ii)

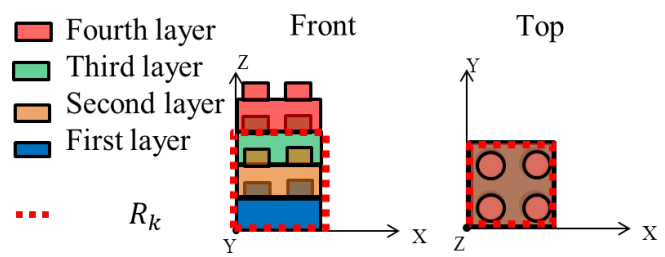

(b) The top block fulfills condition (iii)

Figure 4. Case stable.
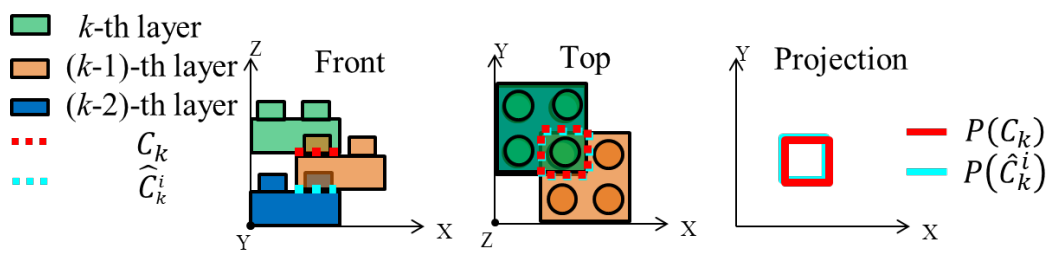

Figure 5. Case inclusion.

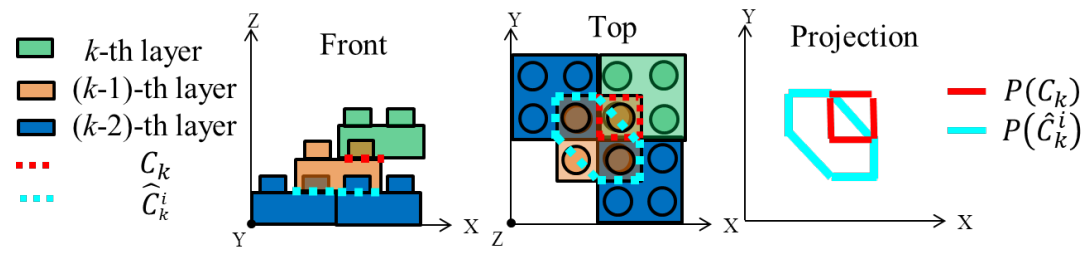

Figure 6. Case partial inclusion. 


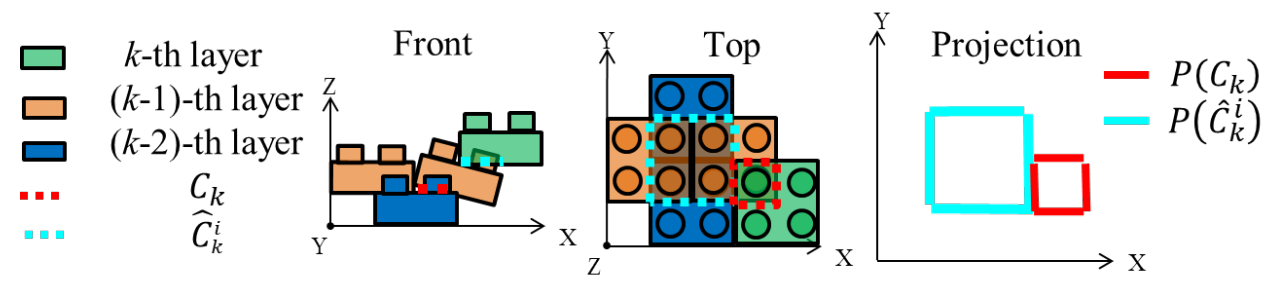

(a) The green block is not assemblable without adjacent block.

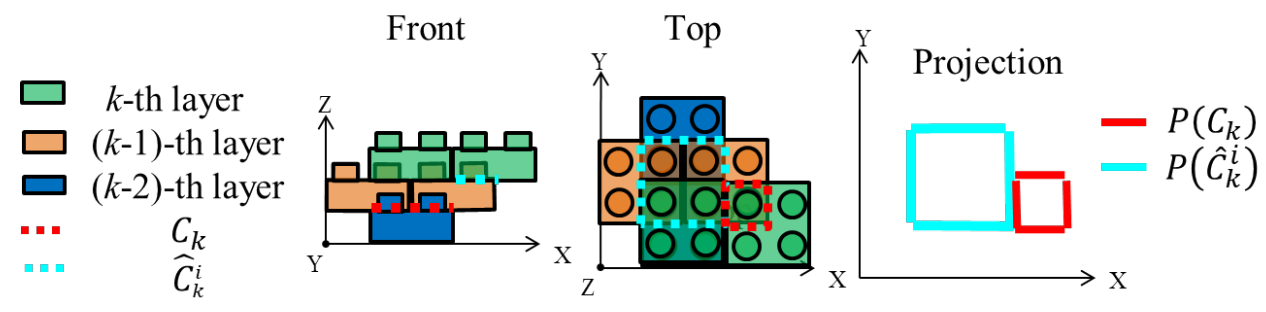

(b) The right green block become assemblable due to an adjacent block (the left green block).

Figure 7. Case adjacent support.

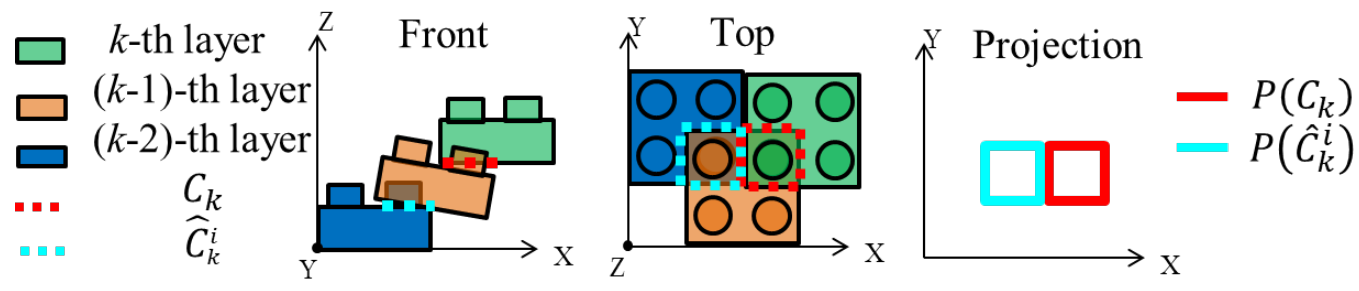

(a) $P\left(C_{k}\right)$ shares no region with $P\left(\hat{C}_{k}^{i}\right)$.

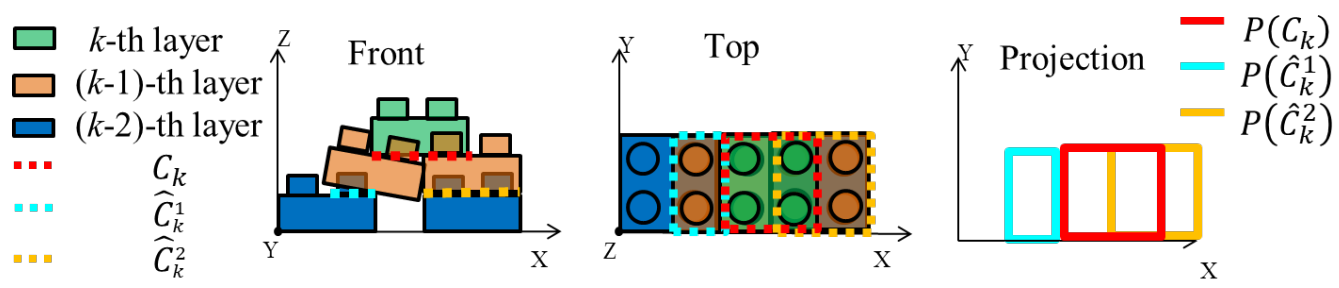

(b) $P\left(\hat{C}_{k}^{1}\right)$ does not share region with $P\left(C_{k}\right)$ though $P\left(\hat{C}_{k}^{2}\right)$ does.

Figure 8. Case unassemblable. 


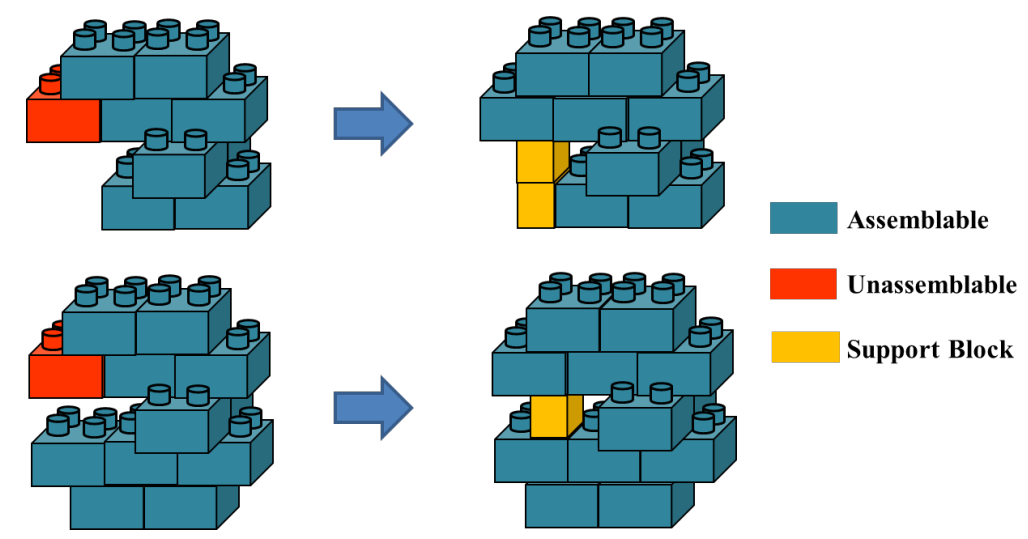

Figure 9. Adding support blocks for unassemblable blocks.
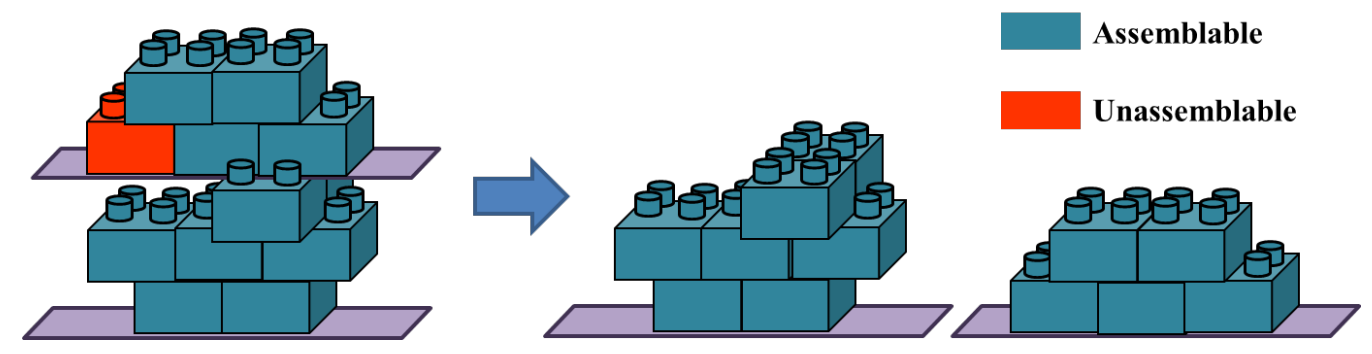

Figure 10. Dividing into subassemblies.

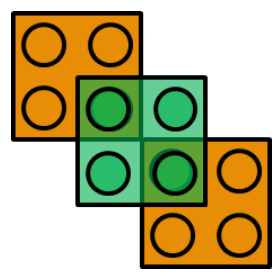

(a) $n_{\text {block }}=2, n_{\text {stud }}=2$

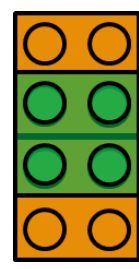

(b) $n_{\text {block }}=2, n_{\text {stud }}=4$

Figure 11. Block indices of the green blocks. 


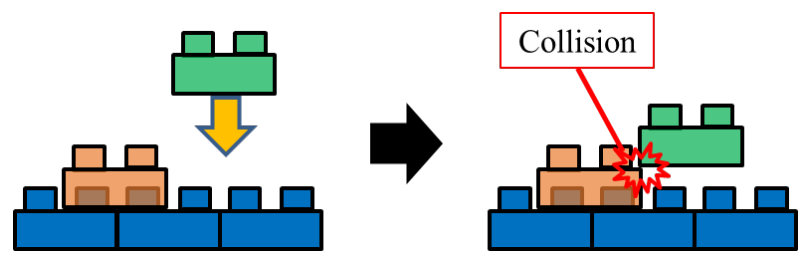

Figure 12. Vertical placement can cause collision between the edges of the blocks.

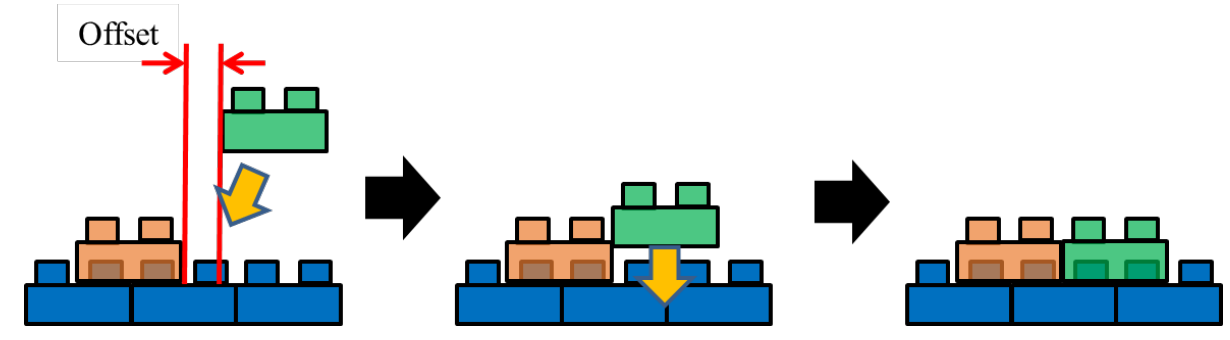

Figure 13. Inclined placement avoids collision. It raises success probability. 


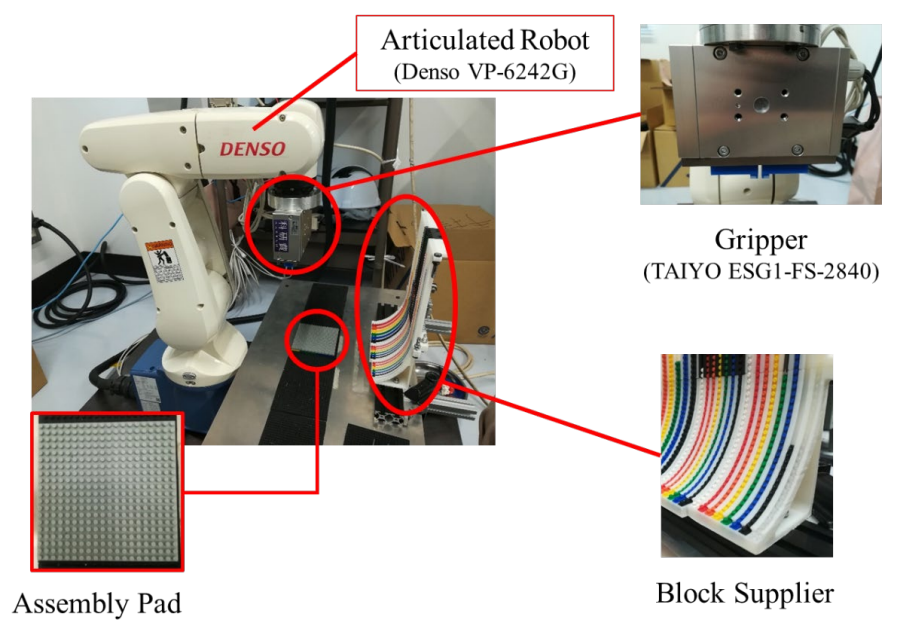

Figure 14. Experimental setup. The robot picks a block from the bottom of the block supplier according to the placement order. The block is placed to the assembly pad.
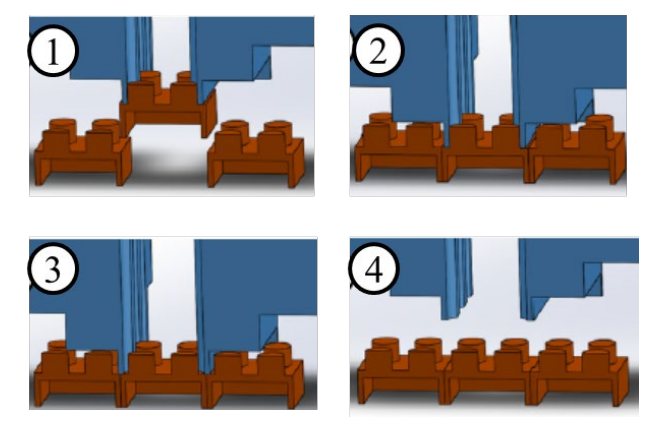

Figure 15. Grasp studs of a nanoblock to carry it (Maeda et al., 2016). 


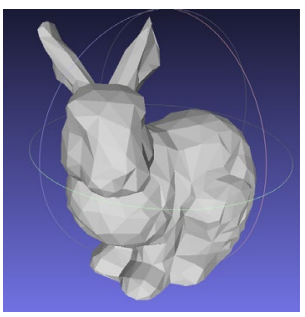

(a) CAD model* ${ }^{*}$

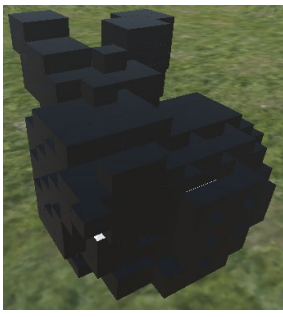

(b) Block model

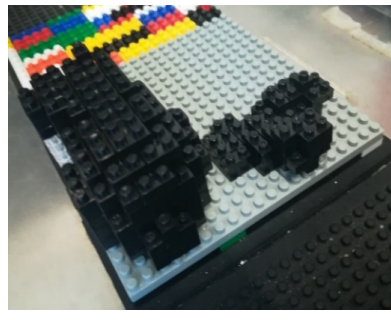

(c) Subassemblies

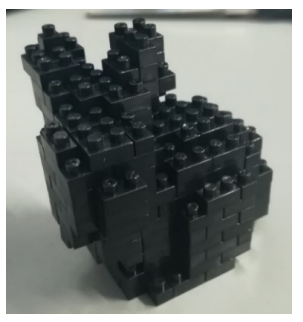

(d) Final assembly

* (Thingiverse 2019A)

Figure 16. A bunny.

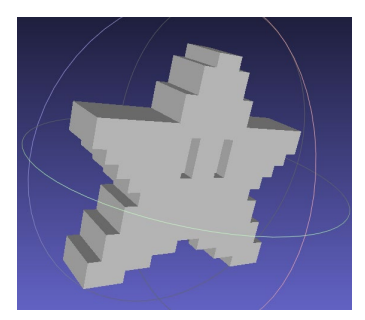

(a) CAD model*.

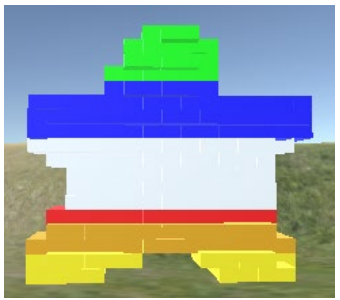

(b) Block model

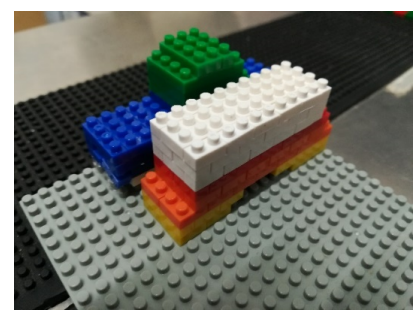

(c) Subassemblies

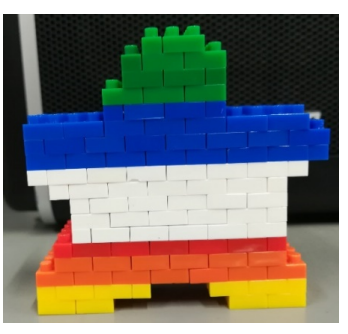

(d) Final assembly

* (Thingiverse 2019B)

Figure 17. A star.

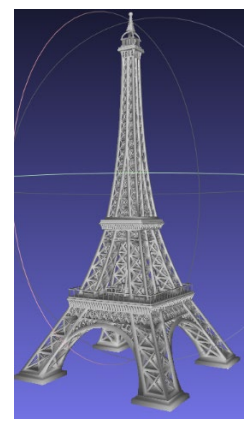

(a) CAD model*

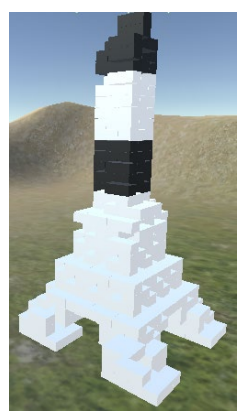

(b) Block model

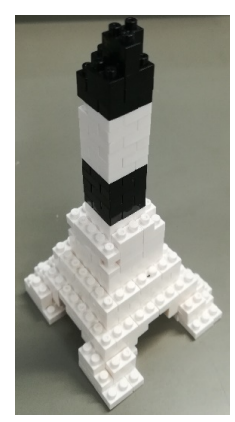

(c) Final assembly

* (Thingiverse 2019C)

Figure 18. A tower. 


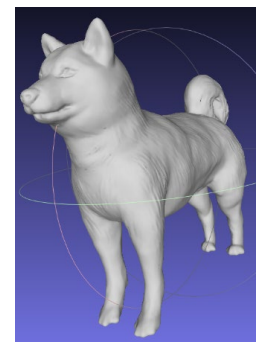

(a) CAD model* .

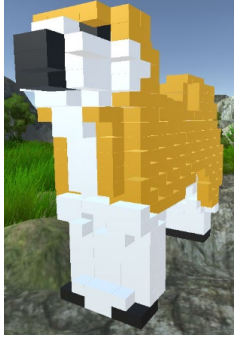

(b) Block model

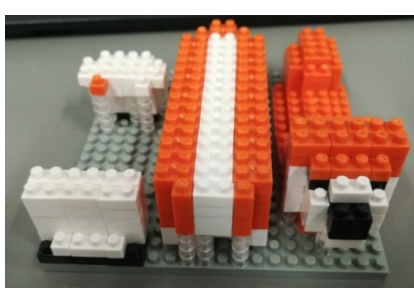

(c) Subassemblies

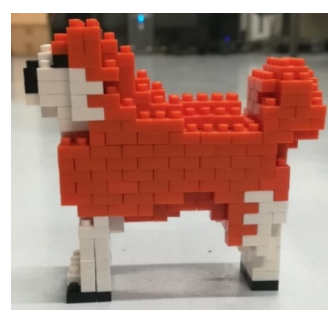

(d) Final assembly

* (Thingiverse 2019D)

Figure 19. A dog.
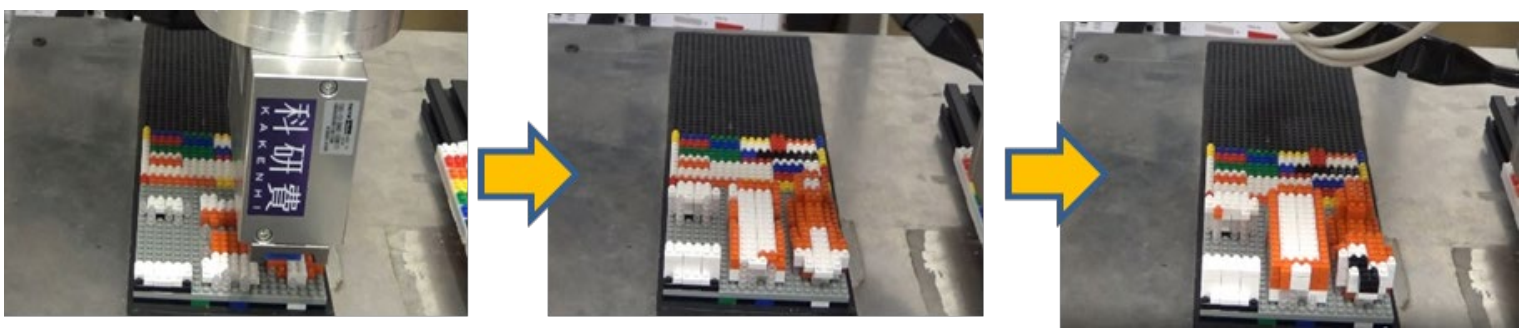

Figure 20. A scene in dog model assembly. 